\title{
On the entangled ergodic theorem
}

\author{
TANJA EISNER AND DÁVID KunsZENTI-KovÁCS
}

\begin{abstract}
We study the convergence of the so-called entangled ergodic averages

$$
\frac{1}{N^{k}} \sum_{n_{1}, \ldots, n_{k}=1}^{N} T_{m}^{n_{\alpha(m)}} A_{m-1} T_{m-1}^{n_{\alpha(m-1)}} A_{m-2} \ldots A_{1} T_{1}^{n_{\alpha(1)}},
$$

where $k \leq m$ and $\alpha:\{1, \ldots, m\} \rightarrow\{1, \ldots, k\}$ is a surjective map. We show that, on general Banach spaces and without any restriction on the partition $\alpha$, the above averages converge strongly as $N \rightarrow \infty$ under some quite weak compactness assumptions on the operators $T_{j}$ and $A_{j}$. A formula for the limit based on the spectral analysis of the operators $T_{j}$ and the continuous version of the result are presented as well.
\end{abstract}

Mathematics Subject Classification (2010): 47A35 (primary); 37A30 (secondary).

\section{Introduction}

The classical mean ergodic theorem has inspired many mathematicians and led to several generalisations and extensions. We mention Berend, Lin, Rosenblatt, Tempelman [3] for modulated and subsequential ergodic theorems and e.g. Kra [13] for an overview on multiple ergodic theorems as well as for the history of the subjects and further references.

In this note we study a further extension of the mean ergodic theorem, namely the so-called entangled ergodic theorem. Let $\alpha:\{1, \ldots, m\} \rightarrow\{1, \ldots, k\}$ be a surjective map for some positive integers $k \leq m$, and take $T_{1}, \ldots, T_{m}$ and $A_{1}, \ldots, A_{m-1}$ to be linear operators on a Banach space $X$. We investigate the convergence of the entangled Cesàro means

$$
\frac{1}{N^{k}} \sum_{n_{1}, \ldots, n_{k}=1}^{N} T_{m}^{n_{\alpha(m)}} A_{m-1} T_{m-1}^{n_{\alpha(m-1)}} A_{m-2} \ldots A_{1} T_{1}^{n_{\alpha(1)}} .
$$

This type of ergodic theorems was introduced by Accardi, Hashimoto, Obata [1] motivated by quantum stochastics and was then studied by Liebscher [15] and 
F. Fidaleo $[9,11]$. In their studies, $T_{1}=\ldots=T_{m}=: U$ is a unitary operator on a Hilbert space. Besides a technical assumption of Liebscher [15], there were two basic situations in which weak or strong convergence of the entangled ergodic averages could be proved. The first is when $A_{j}$ are arbitrary and the unitary operator $U$ is almost periodic (see Definition 2.1 below), see Liebscher [15] and Fidaleo [11]. The second is when the operators $A_{j}$ are compact and $U$ is arbitrary unitary, see Fidaleo [9].

In this paper we consider a more general situation. We assume the operators $T_{j}$ belong to a large class including power bounded operators on reflexive Banach spaces. Further, we require a quite general compactness condition on the pairs $\left(A_{j}, T_{j}\right)$ generalising both of the above cases. More precisely, we make the following assumptions.

(A1) (Weakly compact orbits of $T_{j}$ )

The operator $T_{m}$ is power bounded and totally mean ergodic and each of $T_{1}, \ldots, T_{m-1}$ has relatively weakly compact orbits, i.e., $\left\{T_{j}^{n} x: n \in \mathbb{N}\right\}$ is relatively compact in $X$ in the weak topology for every $x \in X$ and $1 \leq j \leq m-1$.

(A2) (Joint compactness of $\left(A_{j}, T_{j}\right)$ )

Every $A_{j}$ is compact on the orbits of $T_{j}$, i.e., $\left\{A_{j} T_{j}^{n} x: n \in \mathbb{N}\right\}$ is relatively compact in $X$ for every $x \in X$ and $1 \leq j \leq m-1$.

Recall that an operator $T$ is called power bounded if $\sup _{n \in \mathbb{N}}\left\|T^{n}\right\|<\infty$. Moreover, $T$ is called totally mean ergodic if the operators $\lambda T$ are mean ergodic for every $\lambda \in \mathbb{T}, \mathbb{T}$ the unit circle. Every operator with relatively weakly compact orbits is automatically totally mean ergodic. Note that assumption (A1) is not very restrictive. As mentioned above, every power bounded operator on a reflexive Banach space has relatively weakly compact orbits by the Banach-Alaoglu theorem. Another important class of examples is given by power bounded positive operators on a Banach lattice $L^{1}(\mu)$ preserving an order interval generated by a strictly positive function, see e.g. Schaefer [18, Theorem II.5.10(f) and Proposition II.8.3] or [7, Section I.1] for further information. While forming a large class, operators with relatively weakly compact orbits admit good asymptotic properties, see Theorem 3.1 below.

Under the assumptions (A1) and (A2) we show that the entangled Cesàro means (1.1) converge strongly and describe their limit operator, see Theorem 3.4. It turns out that only specially interacting projections corresponding to unimodular eigenvalues of $T_{j}$ (in combination with the operators $A_{j}$ ) contribute to the limit.

The paper is organised as follows. We first treat the special case assuming that all but the last $T_{j}$ are almost periodic (Section 2). Here we show how to reduce the problem to the case when $T_{1}=\ldots=T_{m-1}$ and $A_{1}=\ldots=A_{m-1}$. In the context of Hilbert spaces and pair partitions, this case was considered recently in Fidaleo [11]. In Section 3 the general case is treated. We further discuss the connection to noncommutative multiple ergodic theorems, them being an important recent field of 
research. In Section 5, we finally study the case of strongly continuous semigroups and the strong convergence of the ergodic averages

$$
\frac{1}{t^{k}} \int_{[0, t]^{k}} T_{m}\left(s_{\alpha(m)}\right) A_{m-1} T_{m-1}\left(s_{\alpha(m-1)}\right) A_{m-2} \ldots A_{1} T_{1}\left(s_{\alpha(1)}\right) d s_{1} \ldots d s_{k} .
$$

Note that the study of convergence of entangled ergodic averages for the continuous time scale seems to be new.

Before proceeding we first show by an example that one cannot drop assumption (A2) even when $m=2$ and the operator $T_{1}=T_{2}=: U$ is unitary and weakly stable, i.e., satisfies $\lim _{n \rightarrow \infty} U^{n}=0$ in the weak operator topology.

Proposition 1.1. There exists a Hilbert space $H$, a weakly stable unitary operator $U$ on $H$ and an operator $A \in \mathcal{L}(H)$ such that the Cesàro means

$$
\frac{1}{N} \sum_{n=1}^{N} U^{n} A U^{n}
$$

do not converge weakly.

Proof. Let $H:=\ell^{2}(\mathbb{Z})$ and consider the standard orthonormal base $\left\{e_{b}\right\}_{b \in \mathbb{Z}}$. Let then $U$ be the unitary left shift operator. Let further $(f(n))_{n \in \mathbb{N}^{+}}$be a $0-1$ sequence that is not Cesàro-summable, and define $A$ by

$$
A e_{b}:= \begin{cases}e_{f(-b)-b} & \text { whenever } b<0 \\ e_{b} & \text { whenever } b \geq 0 .\end{cases}
$$

Then $A$ is a bounded operator, and we have $U^{n} A U^{n} e_{0}=e_{f(n)}$ for all $n \in \mathbb{N}$. Hence $\left\langle U^{n} A U^{n} e_{0}, e_{0}\right\rangle=1-f(n)$, and since $(f(n))_{n \in \mathbb{N}^{+}}$is not Cesàro-summable, the Cesàro-means $\frac{1}{N} \sum_{n=1}^{N} U^{n} A U^{n}$ cannot converge weakly either.

ACKNOWLEDGements. The authors are grateful to Rainer Nagel and Marco Schreiber for valuable discussions and comments.

\section{The almost periodic case}

We first consider the situation when all operators $T_{j}$ are almost periodic. In the case when $\alpha$ is a pair partition, $X$ is a Hilbert space and the operators $T_{j}$ are all equal to a unitary operator, this has been studied by Liebscher [15] and Fidaleo [11].

Definition 2.1. An operator $T \in \mathcal{L}(X)$ acting on a Banach space is called almost periodic if it is power bounded and satisfies

$$
X=\varlimsup \operatorname{lin}\{x \in X \mid \exists \lambda \in \mathbb{T}: T x=\lambda x\} .
$$


The following result is the first step towards the general situation.

Theorem 2.2. Let $X$ be a Banach space, $T_{1}, \ldots, T_{m-1}$ be almost periodic operators on $X, T_{m} \in \mathcal{L}(X)$ be power bounded and totally mean ergodic, $A_{1}, \ldots, A_{m-1} \in$ $\mathcal{L}(X)$, and $\alpha:\{1, \ldots, m\} \rightarrow\{1, \ldots, k\}$ be surjective for some $k \leq m$. Then the entangled Cesàro means

$$
\frac{1}{N^{k}} \sum_{n_{1}, \ldots, n_{k}=1}^{N} T_{m}^{n_{\alpha(m)}} A_{m-1} T_{m-1}^{n_{\alpha(m-1)}} A_{m-2} \ldots A_{1} T_{1}^{n_{\alpha(1)}}
$$

converge strongly as $N \rightarrow \infty$.

Proof. We shall proceed by induction on $m$, giving an explicit form for the limit.

First recall that any operator with relatively weakly compact orbits (in particular any almost periodic operator) is mean ergodic, see e.g. [7, Theorem I.2.9], and to have relatively weakly compact orbits is an invariant property under multiplication by a unimodular constant. We may therefore introduce the mean ergodic projections

$$
P_{\lambda}^{(j)}:=\lim _{N \rightarrow \infty} \frac{1}{N} \sum_{n=1}^{N}\left(\lambda^{-1} T_{j}\right)^{n},
$$

where $1 \leq j \leq m$ and $|\lambda|=1$

We now show that the limit of the entangled Cesàro means (2.1) is given by the formal sum

$$
\sum_{\substack{\lambda_{j} \in \sigma_{j}(1 \leq j \leq m) \\ \prod_{i \in \alpha^{-1}(a)} \lambda_{i}=1(1 \leq a \leq k)}} P_{\lambda_{m}}^{(m)} A_{m-1} P_{\lambda_{m-1}}^{(m-1)} A_{m-2} \ldots A_{1} P_{\lambda_{1}}^{(1)} .
$$

Here, $\sigma_{j}$ denotes the point spectrum of $T_{j}(1 \leq j \leq m)$ and (2.2) should be understood as the strong limit of the net

$$
\left\{\sum_{\substack{\lambda_{j} \in F_{j}(1 \leq j \leq m) \\ \prod_{i \in \alpha^{-1}(a)} \lambda_{i}=1(1 \leq a \leq k)}} P_{\lambda_{m}}^{(m)} A_{m-1} P_{\lambda_{m-1}}^{(m-1)} A_{m-2} \ldots A_{1} P_{\lambda_{1}}^{(1)}\right\}_{F_{j} \subset \sigma_{j} \text { finite }(1 \leq j \leq m)}
$$

As seen above, this holds for $m=1$. Suppose we know that it holds for any choice of $(m-2)$ almost periodic operators $T_{j}$ and $(m-2)$ bounded operators $A_{i}(m \geq 2)$. We may suppose without loss of generality that $\alpha(1)=1$.

If now $\alpha^{-1}(1)=\{1\}$, then (2.1) can be written as

$$
\left(\frac{1}{N^{(k-1)}} \sum_{n_{2}, \ldots, n_{k}=1}^{N} T_{m}^{n_{\alpha(m)}} A_{m-1} T_{m-1}^{n_{\alpha(m-1)}} A_{m-2} \ldots A_{2} T_{2}^{n_{\alpha(2)}}\right) A_{1}\left(\frac{1}{N} \sum_{n_{1}=1}^{N} T_{1}^{n_{1}}\right) .
$$


By the induction hypotheses and the joint continuity of multiplication in the strong operator topology, this expression converges to

$$
\begin{aligned}
& \left(\sum_{\substack{\lambda_{j} \in \sigma_{j}(2 \leq j \leq m) \\
\prod_{i \in \alpha^{-1}(a)} \lambda_{i}=1(2 \leq a \leq k)}} P_{\lambda_{m}}^{(m)} A_{m-1} P_{\lambda_{m-1}}^{(m-1)} A_{m-2} \ldots A_{2} P_{\lambda_{2}}^{(2)}\right) A_{1} P_{1}^{(1)} \\
& =\sum_{\substack{\lambda_{j} \in \sigma_{j}(1 \leq j \leq m) \\
\prod_{i \in \alpha^{-1}(a)} \lambda_{i}=1(1 \leq a \leq k)}} P_{\lambda_{m}}^{(m)} A_{m-1} P_{\lambda_{m-1}}^{(m-1)} A_{m-2} \ldots A_{1} P_{\lambda_{1}}^{(1)} .
\end{aligned}
$$

If, on the other hand, there exists an $1<l \leq m$ with $\alpha(l)=1$, then consider an eigenvector $x \in X$ of $T_{1}$ pertaining to some eigenvalue $\lambda \in \mathbb{T}$. We can rewrite the entangled means applied to $x$ as

$$
\begin{aligned}
& \frac{1}{N^{k}} \sum_{n_{1}, \ldots, n_{k}=1}^{N} T_{m}^{n_{\alpha(m)}} A_{m-1} T_{m-1}^{n_{\alpha(m-1)}} A_{m-2} \ldots A_{1} T_{1}^{n_{\alpha(1)}} x \\
= & \frac{1}{N^{k}} \sum_{n_{1}, \ldots, n_{k}=1}^{N} T_{m}^{n_{\alpha(m)}} A_{m-1} T_{m-1}^{n_{\alpha(m-1)}} A_{m-2} \ldots A_{1} \lambda^{n_{\alpha(1)}} x \\
= & \frac{1}{N^{k}} \sum_{n_{1}, \ldots, n_{k}=1}^{N} T_{m}^{n_{\alpha(m)}} A_{m-1} T_{m-1}^{n_{\alpha(m-1)}} A_{m-2} \ldots\left(\lambda T_{l}\right)^{n_{\alpha(l)}} \ldots A_{2} T_{2}^{n_{\alpha(2)}}\left(A_{1} x\right) .
\end{aligned}
$$

This reduces the problem to the $(m-2)$ almost periodic operators $T_{2}, \ldots, \lambda T_{l}, \ldots, T_{m}$, and $(m-2)$ bounded operators $A_{2}, \ldots, A_{m-1}$. The induction hypotheses together with $x=P_{\lambda}^{(1)} x$ yields that the limit is

$$
\begin{aligned}
& \sum_{\substack{\lambda_{j} \in \sigma_{j}(2 \leq j \leq m, j \neq l) \\
\lambda_{l} \in \lambda \sigma_{l} \\
\prod_{i \in \alpha^{-1}(a) \backslash\{1\}} \lambda_{i}=1(1 \leq a \leq k)}} P_{\lambda_{m}}^{(m)} A_{m-1} P_{\lambda_{m-1}}^{(m-1)} A_{m-2} \ldots P_{\lambda^{-1} \lambda_{l}}^{(l)} \ldots A_{2} P_{\lambda_{2}}^{(2)}\left(A_{1} x\right) \\
&=\sum_{\substack{\lambda_{j} \in \sigma_{j}(2 \leq j \leq m) \\
\lambda_{1}=\lambda}}^{\prod_{i \in \alpha^{-1}(a)}^{\lambda_{i}=1(1 \leq a \leq k)}} \\
&=\sum_{\lambda_{\lambda_{m}}^{(m)}} A_{m-1} P_{\lambda_{m-1}}^{(m-1)} A_{m-2} \ldots P_{\lambda_{l}}^{(l)} \ldots A_{2} P_{\lambda_{2}}^{(2)} A_{1} P_{\lambda_{1}}^{(1)} x \\
& \prod_{\substack{\lambda_{j} \in \sigma_{j}\left(1 \leq j \leq \alpha^{-1}(a) \\
\lambda_{i}=1(1 \leq a \leq k)\right.}} P_{\lambda_{m}}^{(m)} A_{m-1} P_{\lambda_{m-1}}^{(m-1)} A_{m-2} \ldots A_{1} P_{\lambda_{1}}^{(1)} x .
\end{aligned}
$$

Since the eigenvectors of $T_{1}$ corresponding to unimodular eigenvalues span $X$ and the entangled Cesàro means are uniformly bounded, we obtain the convergence on $X$ to the required limit. 
The following proposition shows that for the study of convergence of the entangled ergodic averages, it may be assumed without loss of generality that all operators $A_{j}$ are equal, and the same for the operators $T_{j}(1 \leq j \leq m-1)$. This trick will be used later, to simplify the proofs of Theorem 3.4 and Theorem 5.5.

Proposition 2.3. Suppose we know that Theorem 2.2 and the form of the limit given by (2.2) hold under additional assumption $T_{1}=\ldots=T_{m-1}$ and $A_{1}=\ldots=$ $A_{m-1}$. Then it also holds in full generality.

Proof. Consider the space $\mathcal{X}:=X^{m}$ with the diagonal operators

$$
\begin{aligned}
\mathcal{T} & :=\operatorname{diag}\left(T_{1}, T_{2}, \ldots, T_{m-1}, I\right) \in \mathcal{L}(\mathcal{X}), \\
\mathcal{S} & :=\operatorname{diag}\left(I, I, \ldots, I, T_{m}\right) \in \mathcal{L}(\mathcal{X})
\end{aligned}
$$

and the off-diagonal operator

$$
\mathcal{A}:=\left(\left(\delta_{a-1, b} A_{a}\right)\right)_{a, b} \in \mathcal{L}(\mathcal{X}),
$$

where $\delta$ is the Kronecker symbol. Since all the $T_{j}$ 's $(1 \leq j \leq m-1)$ are almost periodic, so is $\mathcal{T}$, and $\mathcal{S}$ is clearly totally mean ergodic on $\mathcal{X}$. We can hence apply the weaker statement of Theorem 2.2 to the operators $\mathcal{T}, \mathcal{S}$ and $\mathcal{A}$. For the vector $(x, 0, \ldots, 0)^{T} \in \mathcal{X}$ for some $x \in X$, this yields the existence of

$$
\lim _{N \rightarrow \infty} \frac{1}{N^{k}} \sum_{n_{1}, \ldots, n_{k}=1}^{N} \mathcal{S}^{n_{\alpha(m)}} \mathcal{A} \mathcal{T}^{n_{\alpha(m-1)}} \mathcal{A} \ldots \mathcal{A T}^{n_{\alpha(1)}}(x, 0, \ldots, 0)^{T} .
$$

However, each of the summands has the form

$$
\left(0, \ldots, 0, T_{m}^{n_{\alpha(m)}} A_{m-1} T_{m-1}^{n_{\alpha(m-1)}} A_{m-2} \ldots A_{1} T_{1}^{n_{\alpha(1)}} x\right)^{T},
$$

and hence the convergence of (2.3) in the last coordinate implies the required convergence of (2.1). Concerning the explicit form of the limit in question, the expression (2.2) yields that it is the last coordinate of

$$
\sum_{\substack{\lambda_{j} \in \sigma(1 \leq j \leq m) \\ \prod_{i \in \alpha^{-1}(a)}}} \mathcal{P}_{\lambda_{m}=1(1 \leq a \leq k)}^{\mathcal{S}} \mathcal{A} \mathcal{P}_{\lambda_{m-1}}^{\mathcal{T}} \ldots \mathcal{A} \mathcal{P}_{\lambda_{1}}^{\mathcal{T}}(x, 0, \ldots, 0)^{T},
$$

where $\mathcal{P}_{\lambda}^{\mathcal{S}}$ and $\mathcal{P}_{\lambda}^{\mathcal{T}}$ are the mean ergodic projections onto the eigenspace corresponding to $\lambda$ of $\mathcal{S}$ and $\mathcal{T}$, respectively. Due to the diagonality of $\mathcal{T}$ and $\mathcal{S}$, each of the components in $\mathcal{X}=X^{m}$ is $\mathcal{T}$ - and $\mathcal{S}$-invariant, hence we in fact have $\mathcal{P}_{\lambda}^{\mathcal{T}}=$ $\operatorname{diag}\left(P_{\lambda}^{(1)}, \ldots, P_{\lambda}^{(m-1)}, \mathbb{1}_{\{1\}}(\lambda) I\right)$ and $\mathcal{P}_{\lambda}^{\mathcal{S}}=\operatorname{diag}\left(\mathbb{1}_{\{1\}}(\lambda) I, \ldots, \mathbb{1}_{\{1\}}(\lambda) I, P_{\lambda}^{(m)}\right)$ where $\mathbb{1}_{M}$ denotes the charasteristic function of a set $M$. The summands in (2.4) thus have the form

$$
\left(0, \ldots, 0, P_{\lambda_{m}}^{(m)} A_{m-1} P_{\lambda_{m-1}}^{(m-1)} A_{m-2} \ldots A_{1} P_{\lambda_{1}}^{(1)} x\right)^{T} .
$$

Taking into consideration that the mean ergodic projections satisfy $\operatorname{Ran} P_{\lambda}^{(j)}=$ $\left\{y \in X \mid T_{j} y=\lambda y\right\}$, and hence $P_{\lambda}^{(j)}=0$ whenever $\lambda \notin \sigma_{j}$, the limit reduces to the required form. 


\section{The general case}

We now extend the results from the previous section to the case when only the orbits along the family of operators $\left\{A_{i} T_{i}^{n}\right\}_{n \in \mathbb{N}^{+}}$are relatively compact for all $1 \leq i \leq$ $m-1$, i.e., assuming (A2).

The key for our considerations will be the following extended version of a classical decomposition theorem, see e.g. Krengel [14, Section 2.2.4] or [7, Theorem II.4.8].

Theorem 3.1 (Jacobs-Glicksberg-de Leeuw decomposition). Let $X$ be a Banach space and let $T \in \mathcal{L}(X)$ have relatively weakly compact orbits. Then $X=X_{r} \oplus X_{s}$, where

$$
\begin{aligned}
& X_{r}:=\varlimsup \\
& X_{s}:=\left\{x \in X \mid \lim _{j \rightarrow \infty} T^{n_{j}} x=0 \text { weakly for some sequence }\left\{n_{j}\right\}_{j=1}^{\infty} \text { with density } 1\right\}
\end{aligned}
$$

with both subspaces being invariant under T. In addition, if $X^{\prime}$ is separable, then there exists a sequence $\left\{n_{j}\right\}_{j=1}^{\infty}$ with density 1 such that $\left.\lim _{j \rightarrow \infty} T^{n_{j}}\right|_{X_{s}}=0$ weakly.

Recall that the density of a set $M \subset \mathbb{N}$ is defined by

$$
d(M)=\lim _{n \rightarrow \infty} \frac{|M \cap\{1, \ldots, n\}|}{n} \leq 1,
$$

whenever the above limit exists.

We further need the following well-known facts.

Lemma 3.2 (Koopman-von Neumann). For a bounded sequence $\left\{a_{n}\right\}_{n=1}^{\infty} \subset[0, \infty)$ the following assertions are equivalent.

(a) $\lim _{n \rightarrow \infty} \frac{1}{n} \sum_{k=1}^{n} a_{k}=0$.

(b) There exists a subsequence $\left\{n_{j}\right\}_{j=1}^{\infty}$ of $\mathbb{N}$ with density 1 such that $\lim _{j \rightarrow \infty} a_{n_{j}}=0$.

We refer to e.g. Petersen [17, page 65] for the proof.

Lemma 3.3. Let $X$ be a Banach space and let $\left\{T_{n}\right\}_{n=1}^{\infty},\left\{S_{n}\right\}_{n=1}^{\infty} \subset \mathcal{L}(X)$. If both $\left\{T_{n} x: n \in \mathbb{N}\right\}$ and $\left\{S_{n} x: n \in \mathbb{N}\right\}$ are relatively compact in $X$ for every $x \in X$, then so is $\left\{T_{n} S_{n} x: n \in \mathbb{N}\right\}$ for every $x \in X$.

Proof. Since compact sets are bounded and by the uniform boundedness principle, there exists $M>0$ such that $\left\|T_{n}\right\| \leq M$ and $\left\|S_{n}\right\| \leq M$ holds for every $n \in \mathbb{N}^{+}$. Take now $x \in X$ and a sequence $\left\{n_{j}\right\} \subset \mathbb{N}$. Then there exists a subsequence $\left\{m_{j}\right\}$ of $\left\{n_{j}\right\}$ such that $\lim _{j \rightarrow \infty} S_{m_{j}} x=y$ for some $y \in X$. Furthermore, there exists a 
subsequence of $\left\{m_{j}\right\}$ which we again denote by $\left\{m_{j}\right\}$ such that $\lim _{j \rightarrow \infty} T_{m_{j}} y=z$ for some $z \in X$. This yields

$$
\left\|T_{m_{j}} S_{m_{j}} x-z\right\| \leq M\left\|S_{m_{j}} x-y\right\|+\left\|T_{m_{j}} y-z\right\| \rightarrow 0 \quad \text { as } j \rightarrow \infty,
$$

proving relative compactness of $\left\{T_{n} S_{n} x: n \in \mathbb{N}\right\}$.

The following is the main result of the paper.

Theorem 3.4. Let $X$ be a Banach space and $\alpha:\{1, \ldots, m\} \rightarrow\{1, \ldots k\}$ be surjective for some $k \leq m$. Let further $T_{1}, \ldots, T_{m}, A_{1}, \ldots, A_{m-1} \in \mathcal{L}(X)$ satisfy assumptions (A1) and (A2). Then the entangled ergodic averages

$$
\frac{1}{N^{k}} \sum_{n_{1}, \ldots, n_{k}=1}^{N} T_{m}^{n_{\alpha(m)}} A_{m-1} T_{m-1}^{n_{\alpha(m-1)}} A_{m-2} \ldots A_{1} T_{1}^{n_{\alpha(1)}}
$$

converge strongly, and their limit is given by

$$
\sum_{\substack{\lambda_{j} \in \sigma_{j}(1 \leq j \leq m) \\ l_{i \in \alpha^{-1}(a)} \lambda_{i}=1(1 \leq a \leq k)}} P_{\lambda_{m}}^{(m)} A_{m-1} P_{\lambda_{m-1}}^{(m-1)} A_{m-2} \ldots A_{1} P_{\lambda_{1}}^{(1)}
$$

where $\sigma_{j}=P_{\sigma}\left(T_{j}\right) \cap \mathbb{T}$ and $P_{\lambda_{j}}^{(j)}$ is the projection onto the eigenspace of $T_{j}$ corresponding to $\lambda_{j}$, i.e., the mean ergodic projection of the operator $\overline{\lambda_{j}} T_{j}$.

Proof. As in the proof of Corollary 2.3 we may assume $T_{j}=T$ and $A_{j}=A$ for some $T, A \in \mathcal{L}(X)$ and all $1 \leq j \leq m-1$. For $x \in X$, we have to show convergence of

$$
\frac{1}{N^{k}} \sum_{n_{1}, \ldots, n_{k}=1}^{N} T_{m}^{n_{\alpha(m)}} A T^{n_{\alpha(m-1)}} A \ldots A T^{n_{\alpha(1)}} x .
$$

By Theorem 3.1, the summands in (3.1) satisfy

$$
\begin{aligned}
T_{m}^{n_{\alpha(m)}} A T^{n_{\alpha(m-1)} A} \ldots & A T^{n_{\alpha(1)}} x \\
= & \sum_{a=1}^{m-1} T_{m}^{n_{\alpha(m)}} A \ldots A T^{n_{\alpha(a)}} P_{s} A T^{n_{\alpha(a-1)}} P_{r} A \ldots A T^{n_{\alpha(1)}} P_{r} x \\
& +T_{m}^{n_{\alpha(m)}} A T^{n_{\alpha(m-1)}} P_{r} A \ldots A T^{n_{\alpha(1)}} P_{r} x,
\end{aligned}
$$

where $P_{r}$ and $P_{s}$ are the projections onto $X_{r}$ and $X_{s}$ pertaining to $T$ from Theorem 3.1 , respectively. By Theorem 2.2, the averages of the second summand above converge to the desired limit. It remains to show that the averages of the first summand converge to 0 , i.e., that for every $x \in X$ and $1 \leq a \leq m-1$ one has

$$
\lim _{N \rightarrow \infty} \frac{1}{N^{k}} \sum_{n_{1}, \ldots, n_{k}=1}^{N} T_{m}^{n_{\alpha(m)}} A T^{n_{\alpha(m-1)}} A \ldots A T^{n_{\alpha(a)}} P_{s} A T^{n_{\alpha(a-1)}} P_{r} A \ldots A T^{n_{\alpha(1)}} P_{r} x=0 .
$$


Consider

$$
K:=\left\{A T^{n_{a-1}} P_{r} A \ldots A T^{n_{1}} P_{r} x \mid n_{a-1}, \ldots, n_{1} \in \mathbb{N}\right\}
$$

which is relatively compact by assumption and Lemma 3.3(a). We now show that the dual space of the smallest $T$-invariant subspace $Y$ containing $K$ is separable. Observe first that $Y=\varlimsup\left\{T^{n} x \mid n \in \mathbb{N}, x \in K\right\}$. We first show that the set $\operatorname{Orb}(K):=\left\{T^{n} x \mid n \in \mathbb{N}, x \in K\right\}$ is relatively weakly compact. Take a sequence $\left\{T^{n_{j}} x_{j}\right\}_{j=1}^{\infty}$ with $x_{j} \in K$ and $n_{j} \in \mathbb{N}$. Since $K$ is relatively compact, there exists a subsequence of $\left\{x_{j}\right\}$ (which we again denote by $\left\{x_{j}\right\}$ ) converging to some $z$. Moreover, since $T$ has relatively weakly compact orbits, there is a subsequence of $\left\{n_{j}\right\}$ (which we again denote by $\left\{n_{j}\right\}$ ) such that $\lim _{j \rightarrow \infty} T^{n_{j}} z=w$ weakly for some $w \in X$. So we have

$$
\left|\left\langle T^{n_{j}} x_{j}-w, y\right\rangle\right| \leq\left|\left\langle T^{n_{j}} x_{j}-T^{n_{j}} z, y\right\rangle\right|+\left|\left\langle T^{n_{j}} z-w, y\right\rangle\right| \rightarrow 0 \quad \forall y \in X^{\prime},
$$

i.e., $\lim _{j \rightarrow \infty} T^{n_{j}} x_{j}=w$ weakly, and therefore $\operatorname{Orb}(K)$ is relatively weakly compact. Since $Y$ is separable, and the weak topology is metrisable on weakly compact subsets of separable spaces (see e.g. Dunford, Schwartz [5, Theorem V.6.3]), the weak topology on $\operatorname{Orb}(K)$ is metrisable. So it is induced by countably many $\left\{y_{n}\right\} \subset Y^{\prime}$, implying separability of $Y^{\prime}$ by $Y=\overline{\operatorname{lin}} \mathrm{Orb}(K)$.

Theorem 3.1 assures now the existence of a sequence $\left\{n_{j}\right\} \subset \mathbb{N}$ with density 1 such that $\lim _{j \rightarrow \infty} T^{n_{j}} P_{s} y=0$ weakly for every $y \in K$. This implies

$$
\lim _{j \rightarrow \infty} A T^{n_{j}} P_{s} y=0 \text { weakly for every } y \in K \text {. }
$$

We now show that

$$
\lim _{j \rightarrow \infty}\left\|A T^{n_{j}} P_{s} y\right\|=0 \quad \text { uniformly in } y \in K
$$

Indeed, assume that for some $y \in K, A T^{n_{j}} P_{s} y$ does not converge strongly to zero. Then there exist $\delta>0$ and a subsequence $\left\{m_{j}\right\}$ of $\left\{n_{j}\right\}$ such that $\left\|A T^{m_{j}} P_{s} y\right\| \geq$ $\delta$ for every $j$. By relative compactness of $\left\{A T^{n} P_{s} y: n \in \mathbb{N}\right\}$, the sequence $\left\{A T^{m_{j}} P_{s} y\right\}_{j=1}^{\infty}$ has a strong accumulation point which by (3.3) must be zero, a contradiction. Thus, $\lim _{j \rightarrow \infty}\left\|A T^{n_{j}} P_{s} y\right\|=0$ for every $y \in K$ and therefore uniform in $y \in K$, since strong convergence in $\mathcal{L}(X)$ implies uniform strong convergence on compact sets. Since the sequence $\left\{n_{j}\right\}$ has density 1, the equation (3.2) follows from (3.4) and Lemma 3.2.

\section{Connection to convergence of multiple ergodic averages}

In this section we discuss connection between the considered above entangled ergodic theorems and the important topic as multiple ergodic averages. The latter is concerned with non-commutative dynamical systems and was studied by Niculescu, 
Ströh and Zsidó [16], Duvenhage [6], Beyers, Duvenhage and Ströh [4], Fidaleo [10], and Austin, Eisner, Tao [2].

We first introduce what we mean by a non-commutative dynamical system and corresponding multiple ergodic averages.

Definition 4.1. A von Neumann (or non-commutative) dynamical system is a triple $(\mathcal{M}, \tau, \beta)$, where $\mathcal{M}$ is a von Neumann algebra, $\tau: \mathcal{M} \rightarrow \mathbb{C}$ is a faithful normal trace, and $\beta: \mathcal{M} \rightarrow \mathcal{M}$ is a $\tau$-preserving $*$-automorphism. We say for $k \in \mathbb{N}$ that the multiple ergodic averages

$$
\frac{1}{N} \sum_{n=1}^{N} \beta^{n}\left(a_{1}\right) \beta^{2 n}\left(a_{2}\right) \ldots \beta^{k n}\left(a_{k}\right)
$$

converge strongly if they converge in the $\tau$-norm defined by $\|a\|_{\tau}:=\sqrt{\tau\left(a a^{*}\right)}$. The averages in (4.1) are called weakly convergent if

$$
\frac{1}{N} \sum_{n=1}^{N} \tau\left(a_{0} \beta^{n}\left(a_{1}\right) \beta^{2 n}\left(a_{2}\right) \cdots \beta^{k n}\left(a_{k}\right)\right)
$$

converges as $N \rightarrow \infty$ for every $a_{0} \in \mathcal{M}$.

We recall that by the Gel'fand-Neumark-Segal theory, $\mathcal{M}$ can be identified with a dense subspace of a Hilbert space, where the Hilbert space $H$ can be obtained as the completion of $\mathcal{M}$ with respect to the $\tau$-norm. Thus, identifying elements of $\mathcal{M}$ with elements in $H$ and by the standard density argument, strong convergence of the multiple ergodic averages (4.1) corresponds to norm convergence in $H$ and weak convergence of (4.1) corresponds to weak convergence in $H$.

Recall further that for the automorphism $\beta$ there exists a unitary operator $u \in$ $\mathcal{L}(H)$ such that $\beta(a)=u a u^{-1}$, see e.g. [12, Proposition 4.5.3]. (Note that $u$ does not necessarily belong to $\mathcal{M}$.) Thus, averages (4.1) take the form

$$
\frac{1}{N} \sum_{n=1}^{N} u^{n} a_{1} u^{n} a_{2} \cdots u^{n} a_{k} u^{-k n},
$$

i.e., are a special case of entangled ergodic averages for the constant partition $\alpha(j)=1$ for every $j \in 1, \ldots, k$ and the operators $u, \ldots, u, u^{-k}$.

It is well-known that strong (weak) topology and strong (weak) operator topology on $\mathcal{M}$ coincide on bounded sets. Therefore, there is a direct correspondence between strong (weak) convergence of multiple ergodic averages (4.1) and strong (weak) operator convergence of the entangled ergodic averages (4.2), cf. also Fidaleo [10].

Proposition 4.2. Let $(\mathcal{M}, \tau, \beta)$ be a von Neumann dynamical system and $H$ and $u$ as above. Let further $a_{1}, \ldots, a_{k} \in \mathcal{M}$. Then the multiple ergodic averages (4.1) converge strongly (weakly) if and only if the entangled averages (4.2) converge in the strong (weak) operator topology. 
As was shown in [2], multiple ergodic averages (4.1) do not converge in general for $k \geq 3$. Theorem 3.4 shows now that for every von Neumann dynamical system there is a class $\mathcal{K}$ depending on the system such that the multiple ergodic averages converge strongly whenever $a_{1}, \ldots, a_{k} \in \mathcal{K}$, and $\mathcal{K}$ can be chosen as the subspace of all elements $a \in \mathcal{M}$ such that $\left\{a u^{n}: n \in \mathbb{N}\right\}$ is relatively compact in $\mathcal{L}(H)$ for the strong operator topology.

Remark 4.3. Note that also more general sequences of powers than arithmetic sequences for the multiple ergodic averages can be treated by a slight modification in the last inductive step of the proof of Theorem 2.2. For instance, consider the averages

$$
\frac{1}{N^{k}} \sum_{n_{1}, \ldots, n_{k}=1}^{N} \beta^{n_{\alpha(1)}}\left(a_{1}\right) \beta^{n_{\alpha(1)}+n_{\alpha(2)}}\left(a_{2}\right) \cdots \beta^{\sum_{j=1}^{m} n_{\alpha(j)}}\left(a_{m}\right) .
$$

These can then be rewritten as

$$
\frac{1}{N^{k}} \sum_{n_{1}, \ldots, n_{k}=1}^{N} u^{n_{\alpha(1)}} a_{1} u^{n_{\alpha(2)}} a_{2} \cdots u^{n_{\alpha(m)}} a_{m} u^{-\sum_{j=1}^{m} n_{\alpha(j)}} .
$$

The last exponent being a sum of $n_{\alpha(j)}$ 's rather than a single one does not matter on the almost weakly stable part, the compactness arguments of the proof of Theorem 3.4 still hold. On the almost periodic part however, suppose $x$ is an eigenvector to the unimodular eigenvalue $\lambda$. Then

$$
\begin{aligned}
& \frac{1}{N^{k}} \sum_{n_{1}, \ldots, n_{k}=1}^{N} u^{n_{\alpha(1)}} a_{1} u^{n_{\alpha(2)}} a_{2} \cdots u^{n_{\alpha(m)}} a_{m} u^{-\sum_{j=1}^{m} n_{\alpha(j)}} x \\
= & \frac{1}{N^{k}} \sum_{n_{1}, \ldots, n_{k}=1}^{N} u^{n_{\alpha(1)}} a_{1} u^{n_{\alpha(2)}} a_{2} \cdots u^{n_{\alpha(m)}} a_{m} \lambda^{-\sum_{j=1}^{m} n_{\alpha(j)}} x,
\end{aligned}
$$

and the powers of the eigenvalue $\lambda$ have to be pulled forward to not only a single operator as in the inductive proof of Theorem 2.2, but distributed amongst all of them to get back to the standard form:

$$
\begin{aligned}
& \frac{1}{N^{k}} \sum_{n_{1}, \ldots, n_{k}=1}^{N} u^{n_{\alpha(1)}} a_{1} u^{n_{\alpha(2)}} a_{2} \cdots u^{n_{\alpha(m)}} a_{m} \lambda^{-\sum_{j=1}^{m} n_{\alpha(j)} x} \\
= & \frac{1}{N^{k}} \sum_{n_{1}, \ldots, n_{k}=1}^{N}\left(\lambda^{-1} u\right)^{n_{\alpha(1)}} a_{1}\left(\lambda^{-1} u\right)^{n_{\alpha(2)}} a_{2} \cdots\left(\lambda^{-1} u\right)^{n_{\alpha(m)}} a_{m} x .
\end{aligned}
$$

Hence the averages (4.3) converge strongly if $\left\{a_{j} u^{n}: n \in \mathbb{N}\right\}$ is relatively compact in $\mathcal{L}(H)$ for the strong operator topology for every $1 \leq j \leq m$. 


\section{Continuous case}

In this section we treat the continuous time scale, where the operators $T_{j}$ and their powers are replaced by strongly continuous $\left(C_{0^{-}}\right)$semigroups $\left(T_{j}(t)\right)_{t \geq 0}$. The study of the continuous version for entangled ergodic averages seems to be new. Some steps in the proofs are similar to the discrete case and will be skipped. For the general theory of strongly continuous semigroups we refer to e.g. Engel, Nagel [8]. For a semigroup $(T(t))_{t \geq 0}$ we often write $T(\cdot)$.

Definition 5.1. A $C_{0}$-semigroup of operators $(T(t))_{t \geq 0} \subset \mathcal{L}(X)$ acting on a Banach space $X$ is called almost periodic if it is bounded (i.e. $\sup _{t>0}\|T(t)\|<\infty$ ) and satisfies

$$
X=\varlimsup\left\{x \in X \mid \exists \varphi \in \mathbb{R}: T(t) x=e^{i \varphi t} x \forall t \geq 0\right\} .
$$

Recall that by the spectral mapping theorem (see e.g. [8, Corollary IV.3.8]), $T(t) x=$ $e^{i \varphi t} x$ for every $t \geq 0$ if and only if $B x=i \varphi x$ for the generator $B$ of $T(\cdot)$.

We again treat the almost periodic case first.

Theorem 5.2. Let $X$ be a Banach space, $T_{1}(\cdot), \ldots, T_{m-1}(\cdot)$ be almost periodic $C_{0^{-}}$ semigroups on $X, T_{m}(\cdot)$ a bounded totally mean ergodic $C_{0}$-semigroup on $X$ and $A_{1}, \ldots, A_{m-1} \in \mathcal{L}(X)$. Then the entangled Cesàro means

$$
\frac{1}{t^{k}} \int_{[0, t]^{k}} T_{m}\left(s_{\alpha(m)}\right) A_{m-1} T_{m-1}\left(s_{\alpha(m-1)}\right) A_{m-2} \ldots A_{1} T_{1}\left(s_{\alpha(1)}\right) d s_{1} \ldots d s_{k}
$$

converge strongly as $t \rightarrow \infty$.

The integrals in (5.1) are defined strongly. Recall that a semigroup $(T(t))_{t \geq 0}$ is called totally mean ergodic if the semigroup $\left(e^{i \varphi t} T(t)\right)_{t \geq 0}$ is mean ergodic for every $\varphi \in \mathbb{R}$.

Proof. Since almost periodic $C_{0}$-semigroups are totally mean ergodic by the standard density argument, we can for each $T_{j}(\cdot)(1 \leq j \leq m)$ define the projections $P_{\varphi}^{(j)}$ as the mean ergodic projections of $\left(e^{-i \varphi t} T_{j}(t)\right)_{t \geq 0}$ with range $\{y \in$ $\left.X \mid T_{j}(t) y=e^{i \varphi t} y \forall t \geq 0\right\}$. Let $\sigma_{j}$ denote the point spectrum of the generator $B_{j}$ of the semigroup $T_{j}(\cdot)$ on $i \mathbb{R}(1 \leq j \leq m)$.

The proof can then be concluded by an induction argument on $m$ analogous to the discrete case showing that the limit of the entangled Cesàro means (5.1) is given by

$$
\sum_{\substack{\varphi_{j} \in \sigma_{j}(1 \leq j \leq m) \\ \sum_{i \in \alpha^{-1}(a)} \varphi_{i}=0(1 \leq a \leq k)}} P_{\varphi_{m}}^{(m)} A_{m-1} P_{\varphi_{m-1}}^{(m-1)} A_{m-2} \ldots A_{1} P_{\varphi_{1}}^{(1)} .
$$


We shall use the following analogue of Theorem 3.1, see e.g. [7, Theorem III.5.7].

Theorem 5.3 (Continuous Jacobs-Glicksberg-de Leeuw decomposition). Let $X$ be a Banach space and let $T(\cdot) \subset \mathcal{L}(X)$ be a $C_{0}$-semigroup with relatively weakly compact orbits, i.e., such that $\{T(t) x: t \in[0, \infty)\}$ is relatively compact in $X$ in the weak topology for every $x \in X$. Then $X=X_{r} \oplus X_{s}$, where

$$
\begin{aligned}
& X_{r}=\varlimsup \\
& X_{s}=\left\{x \in X \mid \lim _{M \ni j \rightarrow \infty} T(t) x=0 \text { weakly for some } M \subset[0, \infty) \text { with density } 1\right\},
\end{aligned}
$$

with both subspaces being invariant under $T$. In addition, if $X^{\prime}$ is separable, then there exists a set $M \subset[0, \infty)$ with density 1 such that $\left.\lim _{M \ni t \rightarrow \infty} T(t)\right|_{X_{s}}=0$ weakly.

The density of a set $M \subset[0, \infty)$ is defined by

$$
d(M)=\lim _{t \rightarrow \infty} \frac{\lambda(M \cap[0, t])}{t} \leq 1,
$$

with $\lambda(\cdot)$ denoting the Lebesgue measure, whenever the above limit exists.

We further need the following continuous version of Lemma 3.2.

Lemma 5.4 (Koopman-von Neumann, continuous version). For a continuous function $f:[0, \infty) \rightarrow[0, \infty)$ the following assertions are equivalent.

(a) $\lim _{t \rightarrow \infty} \frac{1}{t} \int_{[0, t]} f(s) d s=0$.

(b) There exists a subset $M$ of $[0, \infty)$ with density 1 such that $\lim _{s \in M, s \rightarrow \infty} f(s)=0$.

For the proof, which is analogous to the discrete case, see e.g. [7, Lemma III.5.2].

The main result of this section is the following:

Theorem 5.5. Let $X$ be a Banach space, $m \in \mathbb{N}, T_{m}(\cdot), \ldots, T_{m}(\cdot)$ be $C_{0}$-semigroups on $X, A_{1}, \ldots, A_{m-1} \in \mathcal{L}(X)$, and $\alpha:\{1, \ldots, m\} \rightarrow\{1, \ldots, k\}$ be a surjective map for some $k, m \in \mathbb{N}$. Assume the following.

$\left(\mathrm{A} 1_{c}\right)$ The semigroup $T_{m}(\cdot)$ is bounded and totally mean ergodic and $T_{j}(\cdot)$ has relatively weakly compact orbits for every $1 \leq j \leq m-1$.

$\left(\mathrm{A} 2_{c}\right)$ Every $A_{j}$ is compact on the orbits of $T_{j}(\cdot)$, i.e., $\left\{A_{j} T_{j}(t) x: t \in[0, \infty)\right\}$ is relatively compact in $X$ for every $x \in X$ and $1 \leq j \leq m-1$.

Then the entangled ergodic averages

$$
\frac{1}{t^{k}} \int_{[0, t]^{k}} T_{m}\left(s_{\alpha(m)}\right) A_{m-1} T_{m-1}\left(s_{\alpha(m-1)}\right) A_{m-2} \ldots A_{1} T_{1}\left(s_{\alpha(1)}\right) d s_{1} \ldots d s_{k}
$$


converge strongly. Denoting the generator of $T_{j}(\cdot)$ by $B_{j}(1 \leq j \leq m)$, the strong limit is given by the formula

$$
\sum_{\substack{i \varphi_{j} \in \sigma_{j}(1 \leq j \leq m) \\ \sum_{j \in \alpha^{-1}(a)} \varphi_{j}=0(1 \leq a \leq k)}} P_{\varphi_{m}}^{(m)} A_{m-1} P_{\varphi_{m-1}}^{(m-1)} A_{m-2} \ldots A_{1} P_{\varphi_{1}}^{(1)},
$$

where $\sigma_{j}=P_{\sigma}\left(B_{j}\right) \cap i \mathbb{R}$ and $P_{\varphi_{j}}^{(j)}$ is the projection onto the eigenspace of $B_{j}$ corresponding to $i \varphi_{j}$, i.e., the mean ergodic projection of the semigroup $\left(e^{-i \varphi_{j} t} T_{j}(t)\right)_{t \geq 0}$.

Proof. Using the arguments from Proposition 2.3, we may again assume that we have $T_{j}(\cdot)=T(\cdot)$ and $A_{j}=A$ for $1 \leq j \leq m-1$. It is to be shown that

$$
\frac{1}{t^{k}} \int_{[0, t]^{k}} T_{m}\left(s_{\alpha(m)}\right) A T\left(s_{\alpha(m-1)}\right) A \ldots A T\left(s_{\alpha(1)}\right) x d s_{1} \ldots d s_{k}
$$

converges for every $x \in X$. By Theorem 5.3, the integrand can be split with the help of the projections $P_{r}$ and $P_{s}$ onto $X_{r}$ and $X_{s}$, respectively, and we have

$$
\begin{aligned}
& T_{m}\left(s_{\alpha(m)}\right) A T\left(s_{\alpha(m-1)}\right) A \ldots A T\left(s_{\alpha(1)}\right) x \\
= & \sum_{a=1}^{m-1} T_{m}\left(s_{\alpha(m)}\right) A \ldots A T\left(s_{\alpha(a)}\right) P_{s} A T\left(s_{\alpha(a-1)}\right) P_{r} A \ldots A T\left(s_{\alpha(1)}\right) P_{r} x \\
+ & T_{m}\left(s_{\alpha(m)}\right) A T\left(s_{\alpha(m-1)}\right) P_{r} A \ldots A T\left(s_{\alpha(1)}\right) P_{r} x .
\end{aligned}
$$

The integral means of the second term converge by Theorem 5.2 to the desired limit, and so it is enough to show that the rest converges in mean to 0 , i.e. for every $x \in X$ and $1 \leq a \leq m-1$ one has

$\lim _{t \rightarrow \infty} \frac{1}{t^{k}} \int_{[0, t]^{k}} T_{m}\left(s_{\alpha(m)}\right) A \ldots A T\left(s_{\alpha(a)}\right) P_{s} A T\left(s_{\alpha(a-1)}\right) P_{r} A \ldots A T\left(s_{\alpha(1)}\right) P_{r} x=0$.

Consider

$$
K:=\left\{A T\left(s_{a-1}\right) P_{r} A \ldots A T\left(s_{1}\right) P_{r} x \mid s_{a-1}, \ldots, s_{1} \in[0, \infty)\right\} .
$$

This set is relatively compact by Lemma 3.3 and the assumption. As in the discrete case, one can show that the dual space of the smallest $T(\cdot)$-invariant subspace $Y$ containing $K$ is separable. Note that the separability of $Y$ itself follows from the strong continuity of the semigroup, as it yields a dense countable subset

$$
\left\{A T\left(s_{a-1}\right) P_{r} A \ldots A T\left(s_{1}\right) P_{r} x \mid s_{a-1}, \ldots, s_{1} \in[0, \infty) \cap \mathbb{Q}\right\}
$$

of $K$.

Theorem 5.3 then assures the existence of a set $M \subset[0, \infty)$ with density 1 such that

$$
\lim _{s_{j} \in M, s_{j} \rightarrow \infty} T\left(s_{j}\right) P_{s} y=0 \quad \text { weakly for every } y \in K
$$


implying $\lim _{s_{j} \in M, s_{j} \rightarrow \infty} A T\left(s_{j}\right) P_{s} y=0$ weakly for every $y \in K$. Since $\left\{A T(s) P_{s} y\right.$ : $s \in[0, \infty)\}$ is relatively compact, and strong convergence in $\mathcal{L}(X)$ implies uniform strong convergence on compact subsets of $X$, we obtain

$$
\lim _{s_{j} \in M, s_{j} \rightarrow \infty}\left\|A T\left(s_{j}\right) P_{s} y\right\|=0 \quad \text { uniformly in } y \in K .
$$

Since the set $M$ has density 1, the equation (5.4) follows from Lemma 5.4.

Note that, as in the discrete case, the class of semigroups satisfying assumption $\left(\mathrm{A} 1_{c}\right)$ is large including e.g. bounded $C_{0}$-semigroups on reflexive Banach spaces.

\section{References}

[1] L. Accardi, Yu. Hashimoto and N. Obata, Notions of independence related to the free group, Infin. Dimens. Anal. Quantum Probab. Relat. Top. 1 (1998), 201-220.

[2] T. AUSTIN, T. EISNER and T. TAO, Nonconventional ergodic averages and multiple recurrence for von Neumann dynamical systems, Pacific J. Math. 250 (2011), 1-60.

[3] D. Berend, M. Lin, J. Rosenblatt and A. Tempelman, Modulated and subsequential ergodic theorems in Hilbert and Banach spaces, Ergodic Theory Dynam. Systems 22 (2002), 1653-1665.

[4] C. Beyers, R. Duvenhage and A. Ströh, The Szemerédi property in ergodic $W^{*}$ dynamical systems, J. Operator Theory 64 (2010), 35-67.

[5] N. DUNFORD and J. T. Schwartz, "Linear Operators" I., Interscience Publishers, Inc., New York; Interscience Publishers, Ltd., London, 1958.

[6] R. Duvenhage, Bergelson's theorem for weakly mixing $C^{*}$-dynamical systems, Studia Math. 192 (2009), 235-257.

[7] T. EISNER, "Stability of Operators and Operator Semigroups", Operator Theory: Advances and Applications, 209, Birkhäuser Verlag, Basel, 2010.

[8] K.-J. ENGEL and R. NAGEL, "One-parameter Semigroups for Linear Evolution Equations", Graduate Texts in Mathematics, Vol. 194, Springer-Verlag, New York, 2000.

[9] F. Fidaleo, On the entangled ergodic theorem, Infin. Dimens. Anal. Quantum Probab. Relat. Top. 10 (2007), 67-77.

[10] F. FidAlEO, An ergodic theorem for quantum diagonal measures, Infin. Dimens. Anal. Quantum Probab. Relat. Top. 12 (2009), 307-320.

[11] F. FIDALEO, The entangled ergodic theorem in the almost periodic case, Linear Algebra Appl. 432 (2010), 526-535.

[12] R. V. KADISON and J. R. RINGROSE, "Fundamentals of the Theory of Operator Algebras", Vol. I. Academic Press, 1983.

[13] B. KRA, Ergodic methods in additive combinatorics, In: "Additive combinatorics", CRM Proc. Lecture Notes, Vol. 43, Amer. Math. Soc., Providence, RI, 2007, 103-143.

[14] U. KREnGEL, "Ergodic Theorems", de Gruyter Studies in Mathematics, de Gruyter, Berlin, 1985.

[15] V. LieBSCHER, Note on entangled ergodic theorems, Infin. Dimens. Anal. Quantum Probab. Relat. Top. 2 (1999), 301-304.

[16] C. P. NiCUleSCU, A. STRÖH and L. Zsidó, Noncommutative extensions of classical and multiple recurrence theorems, J. Operator Theory 50 (2003), 3-52.

[17] K. Petersen, "Ergodic Theory", Cambridge Studies in Advanced Mathematics, Cambridge University Press, 1983. 
[18] H. H. Schaefer, "Banach Lattices and Positive Operators", Springer-Verlag, 1974.

[19] M. TAKESAKI, "Theory of Operator Algebras I", Springer-Verlag, 1979.

KdV Institute for Mathematics

University of Amsterdam

P.O. Box 94248

1090 GE, Amsterdam, The Netherlands

t.eisner@uva.nl

Institute of Mathematics

University of Tübingen

Auf der Morgenstelle 10

72076 Tübingen, Germany daku@fa.uni-tuebingen.de 